\title{
NCDs in Nepal: Burgeoning Burden amid Low Priority and the Ways Forward
}

\author{
Gautam R $\mathbf{R}^{1}$ \\ 'Master in Public Health Candidate \\ Institute of Medicine, Maharajgunj Medical Campus
}

\begin{abstract}
Non-communicable diseases (NCDs) refer to diseases or conditions that occur in, or are known to affect, individuals over an extensive period of time and for which there are no known causative agents that are transmitted from one affected individual to another. (1) WHO has defined NCDs to include chronic diseases, principally cardiovascular disease, diabetes, cancer, and asthma/chronic pulmonary disease (COPD), in addition to injuries and mental illness. (2) The risk factors for many of these conditions are associated with lifestyle related choices, environmental and genetic factors. The behavioural risk factors, that are common and easily modifiableinclude smoking, alcoholism, low quality diet intakes and physical inactivity and these have been found to have $80 \%$ contribution in the development of NCDs. (3) Behavioral risk-factors alone, however, are inadequate in explaining the current rapid rise of NCDs like diabetes and CVD. The intricate relations of NCD with mother and child health $(\mathrm{MCH})$ have also been recognized recently. $(4,5)$ Metabolic/intermediate risk factors like hypertension, high level of blood glucose and cholesterol and BMI are well known triggers of NCDs. (6)
\end{abstract}

It is well established that NCDs are the leading cause of death in the world, responsible for $63 \%$ of the 57 million deaths that occurred in 2008. The majority of these deaths - 36 million - were attributed to cardiovascular diseases and diabetes, cancers and chronic respiratory diseases and $80 \%$ of these deaths occurred in low and middle income countries. $(7,8)$ Similarly, in 2010, the three leading risk factors for global disease burden were high blood pressure (7.0 percent of global DALYs), tobacco smoking, including second-hand smoke (6.3 percent), and alcohol use (5.5\%). (9) Regional data show that in most of SEARO, communicable diseases have been overpowered by NCDs already where NCDs account for $54 \%$ of total mortality and $44 \%$ of total morbidity. (10)

In terms of the number of lives lost due to ill-health, disability, and early death (DALYs) NCDs (inclusive of injuries) account for $60 \%$ of the total disease burden in Nepal while the remaining $40 \%$ is from communicable diseases, maternal and child health, and nutrition issues all combined. (12) The major NCDs that Nepal has been facing are cardiovascular diseases (CVD), injuries, neuropsychiatric conditions, cancers, and to a more moderate extent, chronic respiratory diseases and diabetes.Trend analyses of the metabolic risk factors in Nepal between 1980 and 2008 have shown a distinct upsurge, with an exception of the mean cholesterol level, in the presence of risk factors like high blood pressure, body mass index and blood glucose level. (13) In government hospitals, $80 \%$ outpatient and $88 \%$ of inpatient attendance is due to morbidity related to NCD. (14) Estimates have also been made that future ageing in Nepal will increase the burden of NCDs as the proportion of the population 65 years and older will rise from $4.2 \%$ in 2000, to $5.8 \%$ in 2025. (6) All these figures point towards an understandable serious burden to our health system which has been struggling with high maternal, newborn and child mortality along with the age-old problems of infectious diseases.

This upsurge in the burden of NCDs has been attributed to changing demographics and lifestyles of the population, which includes rapid urbanization, increased industrialization, rising personal incomes, expanded education and improved health care. (11) Contrary to the previously-held belief that NCDs are inflicted by affluence, evidences show that NCDs are becoming common among the poor and marginalized people both in economically advanced countries as well as low and middle income countries. As a result, it is highly probable that much of the progress made over several decades to improve life expectancy rates among the poor in developing countries through the control of infectious diseases could be reversed by the rise of NCDs. (15)

This burgeoning burden is made more complex because of the dearth of adequate and reliable information on most of the NCDs. The NCD risk factor survey provides estimates of intermediate risk factors such as hypertension (9\%), diabetes (10\%) and obesity (7\%) along with the behavioural risk factors. (16) A few hospital-based information and localized community based surveys and census have helped to some extent in providing some insights into it but the regional and national level estimates are still lacking. But even the meager information available clearly points towards high prevalence of the risk factors with an increasing trend. $(13,16,17)$

There has been a renewed interest worldwide on NCDs and consequently progress in terms of the formulation of action plan for global strategy for the prevention and control of NCDs (18) and regional framework for prevention and control of NCDs in South-East Asian region (10) but this has yet to materialize at the national level.Despite having a draft prepared in 2009 of "National policy, strategy and plan of action for prevention and control of non-communicable disease" (19) it has yet to be approved by the government.The formation of NCD prevention and control committee to implement various NCD related activities, ratification of Framework Convention on Tobacco Control, banning of tobacco advertisement in electronic media and of smoking in public places, development and endorsement of other policies related to NCDs such as Nutrition Policy

CORRESPONDENCE: Rupesh Gautam, Master in Public Health Candidate, Maharajgunj Medical Campus, Institute of Medicine, TU, Kathmandu, Nepal, Email:rupesgautam@gmail.com 
and strategy and Mental Health Policy have been some of the commendable efforts by the government towards prevention and control of NCDs. Similarly, in response to the rising importance of NCDs and injuries in the burden of disease, the government has committed that NHSP-2 will expand prevention activities aimed at reducing the burden of NCDs by encouraging healthier lifestyles. (20)

However, despite all these efforts and actions gaps do exist in policy/strategy development, infrastructure, human resource capacity, financing, surveillance, and implementation of key tobacco policy measures.Nepal does not have a reliable baseline that reflects the status of NCD and its risk factors as the NCDs and their risk factors have not yet been included in the national reporting systemnor do we have a national, population-based NCD registry (except for cancer). The cost and complexity of treatment have limited the service accessibility for majority of the poor Nepalese (21) Little effort has been made at the primary health care level for prevention and control of NCDs and their risk factors, the trainings conducted on NCDs for the health workers have only existed at small-scale, and the budget allocated for NCDs has been very low and is mainly dependent on the tax collected from tobacco and alcohol products. A national essential drugs list has been developed but information on NCD-related drugs is not available. (22)

Prevention and control of NCDs is a multi-faceted and complex task as it is linked directly with the people's behaviours which do not change easily and the cost of not intervening is very high in terms of the burden of the morbidity and mortality. Some of the actions that needs to be undertaken immediately at the governmental level to address this burden include: Finalizing national NCD Policy as the draft NCD policy gives a basis for building strategies, plans and action; Strengthening Tobacco Control Policies for consumption reduction through specific focus on taxation and comprehensive bans on tobacco advertising, promotion and sponsorship; Creation of a national surveillance system of NCDs and the associated risk factors; Training of the health workforce in NCD prevention and control; and setting up of specific financing strategies for access to services and medications for the poor (for example: sin tax).

However, as pointed out by Global strategy for prevention and control of NCD the main challenge in prevention and control lies in the prevention of the emergence of risk factors (18) and that points towards the importance of primordial prevention.

\section{References}

1. Daar AS, Singer PA, Persad DL, Pramming SK, Matthews DR, Beaglehole R, et al. Grand challenges in chronic non-communicable diseases. Nature. 2007 Nov 22;450(7169):494-6

2. WHO. World health report 2002: Reducing risks, promoting healthy life. Geneva: WHO, 2003.

3. WHO, Regional Office of South East Asia. Strengthening Partnerships for Integrated Prevention and Control of Non communicable Diseases: a SEANET-NCD Meeting Chandigarh, India. 15-19 June 2009.

4. Bhattarai MD. Primary Prevention and Control of Type 2 Diabetes and Other Non-Communicable Diseases by Healthy Lifestyle Program. Kathmandu: General Welfare Pratisthan, 2010.

5. Bhattarai, M.D. Three Patterns of Rising type 2 Diabetes Prevalence in the World: Need to Widen the Concept of Prevention in Individuals into Control in the Community. J Nep Med Assoc. 2009; 48: 173-9.

6. World Bank: Capitalizing on the Demographic Transition: Tackling Non-communicable Diseases in South Asia (2011)

7. World development indicators. Washington, DC, International Bank for Reconstruction and Development/The World Bank, 2009.

8. Huber L., (2010) Tobacco Control and the Development Agenda. www.fctc.org.

9. Lancet. Global Burden of Disease 2010. [Cited: January 13, 2013]; Available from:http://www.thelancet.com/themed/global-burden-ofdisease

10. WHO/SEARO, (2006) Regional Framework for NCD, New Delhi.

11. Thandi P, Lungiswa T, David S, Whadiah P. Chronic non communicable diseases. School of Public Health, University of the Western Cape and South African Medical Research Council.

12. U.S Census Bureau.[Cited: January 9, 2013]; Available from: www.census.gov/ipc

13. WHO. Non-communicable diseases country profile 2011. [Cited: January 9, 2013]; Available from: whqlibdoc.who.int/publications/ 2011/9789241502283_eng.pdf

14. DoHS, (2012). Annual Report - Department of Health Services (2010/11). Kathmandu: Department of Health services

15. UNESCAP (2009). Non-communicable diseases and achieving the Millennium Development Goals. Bangkok.

16. Ministry of Health and Population, Government of Nepal, Society for Local Integrated Development Nepal (SOLID Nepal) and WHO WHO STEPS Surveillance: Non-Communicable Diseases Risk Factors Survey. Kathmandu: Ministry of Health and Population, Government of Nepal, Society for Local Integrated Development Nepal (SOLID Nepal) and WHO, 2008.

17. Maskey M. Non-communicable Diseases: Emerging Trends and Socio-economic impact.

18. WHO (2008) Action Plan for the Global Strategy for the Prevention and Control of Non communicable Diseases (2008-2013). Geneva.

19. MOHP (2009). Nepal National Policy, Strategy and Plan of Action for Prevention and Control of Non-Communicable Diseases (draft). Government of Nepal. Kathmandu.

20. MOHP. Nepal Health Sector Programme- Implementation Plan II (2010-2015). Kathmandu.

21. Nepal Health Research Council. Prevalence of Non Communicable Disease in Nepal: Hospital Based Study. Kathmandu: Nepal Health Research Council, 2010.

22. WB. (2011) NCD Policy Brief Nepal. [Cited: January 10, 2013]; Available from: http://siteresources.worldbank.org/SOUTHASIAEXT/ Resources/223546-1296680097256/7707437-1296680114157/NCD_NP_Policy_Feb_2011.pdf 